\title{
TESIS experiment on XUV imaging spectroscopy of the Sun onboard the CORONAS-PHOTON satellite
}

\author{
S.V. Kuzin ${ }^{1}$, I.A. Zhitnik ${ }^{1}$, S.A. Bogachev ${ }^{1}$, S.V. Shestov ${ }^{1}$, O.I. \\ Bugaenko $^{2}$, N.K. Suhodrev ${ }^{1}$, A.A. Pertsov ${ }^{1}$, A.V. Mitrofanov ${ }^{1}$, \\ A.P. Ignat'ev ${ }^{1}$, and V.A. Slemzin ${ }^{1}$ \\ ${ }^{1}$ P.N. Lebedev Physical Institute of Russian Academy of Science, Russia \\ email: kuzin@sci.lebedev.ru \\ ${ }^{2}$ P.K. Sternberg Astronomical Institute, Moscow State University, Russia \\ email: bugaenko@sci.lebedev.ru
}

\begin{abstract}
We present a brief description of new complex of space telescopes and spectrographs, TESIS, which will be placed aboard the CORONAS-PHOTON satellite. The complex is intended for high-resolution imaging observation of full Sun in the coronal spectral lines and in the spectral lines of the solar transition region. TESIS will be launched at the end of $2007-$ early of 2008. About $25 \%$ of the daily TESIS images will be free for use and for downloading from the TESIS data center that is planned to open 2 months before the TESIS launching at http://www.tesis.lebedev.ru
\end{abstract}

Keywords. instrumentation: spectrographs, high angular resolution; Sun: transition region, UV radiation, $\mathrm{X}$-rays, gamma rays

\section{Introduction}

In the frameworks of Russian Federal Program of Fundamental Space Researches, the Russian Academy of Science develops space program CORONAS (Complex ORbital ObservatioNs of the Activity of the Sun). From 1993 to 2008, this program assumes the launch of three science satellites adopted for investigation of the Sun and the solarterrestrial connections.

The first satellite of this series, CORONAS-I, was launched in the 1994 March and finished to work six years later in the 2000. Then, in the $2001 \mathrm{July}$, the second satellite, CORONAS-F, was launched to the Earth orbit from the cosmodrome Plesetsk (see Oraevsky \& Sobelman (2002), Zhitnik, Kuzin et al. (2003), Zhitnik, Bugaenko et al. (2003)). The work of the satellite was fulfilled five years later in the January 2006, when the satellite enters the dense Earth's atmosphere.

Now, the third satellite of the CORONAS series, CORONAS-PHOTON, is preparing for launching at the end of 2007 early of 2008. The CORONAS-PHOTON is developed for complex investigation of the solar activity and the solar-terrestrial connections. The main goal of the project is investigation of the high energetic and eruptive process in the solar corona in wide energy range from extreme UV (EUV) up to the high energy gamma radiation $(2000 \mathrm{MeV})$.

Laboratory of X-Ray Astronomy of the Sun (XRAS) of Lebedev Physical Institute of Russian Academy of Science develops the complex of space telescopes and spectrographs TESIS for the CORONAS-PHOTON satellite. This paper gives brief information on the characteristics of TESIS instruments and the objectives of TESIS mission. 

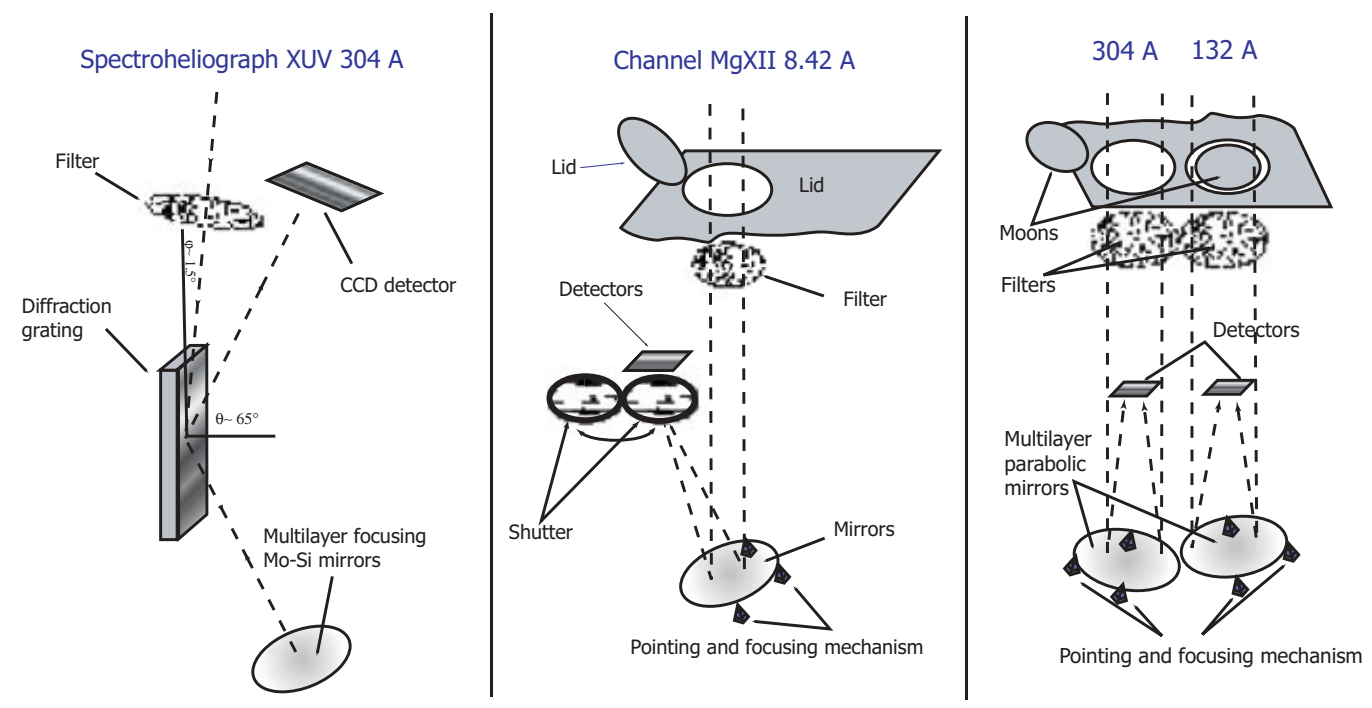

Figure 1. Overview of some TESIS instruments.

\section{TESIS experiment}

At the present time, XRAS laboratory creates new complex of space telescopes and spectrographs, TESIS, which will be placed aboard the CORONAS-PHOTON satellite. The complex is intended for high-resolution imaging observation of full Sun in the coronal spectral lines and in the spectral lines of the solar transition region. TESIS will be the only space instrument, which will provide monochromatic images of the Sun in one spectral line rather than the images obtained in wide spectral range with multiple lines. TESIS will also include a unique imaging spectroheliograph, which gives a set of the simultaneous images of the Sun in the several XUV spectral lines formed at different temperatures (see fig. 1).

All the scientific tasks of the experiment can be divided into three categories: (1) Investigation of the most dynamic processes in the solar atmosphere, such as flares and coronal mass ejections, with the temporal resolution less than $1 \mathrm{sec}$ and the space resolution of about 1-2; (2) Determination of the physical parameters (temperatures, density and differential measures of emission) of hot coronal structures; (3) Study of the largescale coronal magnetic structures: coronal loops, giant magnetic arcades, coronal holes and others.

The total volume of scientific information provided by TESIS is assumed to be about $0.5 \mathrm{~Gb}$ per day, that will be in 10 times more than the volume of information from previous complex, SPIRIT, aboard the CORONAS-F satellite. We expect that TESIS will provide 200-300 high-resolution images of full Sun every day. The size of one decompressed images will be $2048 \times 2048$ pixels with space resolution of 1-3 arcsec for all the TESIS channels, except for coronagraph and spectroheliograph.

About $25 \%$ of the daily TESIS images will be free for use and for downloading from the TESIS data center that is planned to open 2 months before the TESIS launching at http://www.tesis.lebedev.ru 


\section{Conclusions}

We gave a brief description of the instruments and scientific tasks of new complex of space telescopes TESIS, which will be launched at the end of 2007 - early of 2008 onboard the CORONAS-PHOTON satellite. The TESIS will provide 200-300 high-resolution images of full Sun every day. The total volume of scientific information provided by TESIS is assumed to be about $0.5 \mathrm{~Gb}$ per day.

\section{Acknowledgements}

This work is supported by the Russian Foundation of Basic Research under grant 05-02-17415.

\section{References}

Oraevsky, V.N. \& Sobelman, I.I. 2002, Astronomy Letters 28 (6), 401

Zhitnik, I.I., Kuzin, S.V., et al. 2003, Adv. Sp. Res. 32 (12), 2573

Zhitnik, I.I., Bugaenko, O.I., et al. 2003, MNRAS 338 (1), 67 